\title{
A hierarchy of voids
}

\author{
Rien van de Weygaert ${ }^{1}$, Ravi Sheth ${ }^{2,3}$ and Erwin Platen ${ }^{1}$ \\ ${ }^{1}$ Kapteyn Instituut, Rijksuniversiteit Groningen, the Netherlands \\ ${ }^{2}$ Dept. of Physics and Astronomy, University of Pittsburgh, USA \\ ${ }^{3}$ NASA/Fermilab Astrophysics Group, Batavia, IL 60510-0500, USA
}

\begin{abstract}
We present a model for the distribution of void sizes and its evolution within the context of hierarchical scenarios of gravitational structure formation. For a proper description of the hierarchical buildup of the system of voids in the matter distribution, not only the voidin-void problem should be taken into account, but also that of the void-in-cloud issue. Within the context of the excursion set formulation of an evolving void hierarchy is one involving a two-barrier excursion problem, unlike the one-barrier problem for the dark halo evolution. This leads to voids having a peaked size distribution at any cosmic epoch, centered on a characteristic void size that evolves self-similarly in time, in distinct contrast to the distribution of virialized halo masses in not having a small-scale cut-off.
\end{abstract}

\section{Introduction: excursions}

Hierarchical scenarios of structure formation have been very succesfull in understanding the formation histories of gravitationally bound virialized haloes. Particularly compelling has been the formulation of a formalism in which the collapse and virialization of overdense dark matter halos within the context of hierarchical clustering can be treated on a fully analytical basis. This approach was originally proposed by Press \& Schechter (1974), which found a particularly useful and versatile formulation and modification in the excursion set formalism (Bond et al. 1991).

It is based on the assumption that for a structure to reach a particular nonlinear evolutionary stage, such as complete gravitational collapse, the sole condition is that its linearly extrapolated primordial density should attain a certain value. The canonic example is that of a spherical tophat overdensity collapsing once it reaches the collapse barrier $\delta_{c} \approx 1.69$. The successive contributions to the local density by perturbations on a (mass) resolution scale $S_{m}$ may be represented in terms of a density perturbation random walk, the cumulative of all density fluctuations at a resolution scale smaller than $S_{m}$. By identifying the largest scale at which the density passes through the barrier $\delta_{c}$ it is possible (1) to infer at any cosmic epoch the mass spectrum of collapsed halos and (2) to reconstruct the merging history of each halo (see Fig. 3, lefthand). In this study we demonstrate that also the formation and evolution of foamlike patterns as a result of the gravitational growth of primordial density perturbations is liable to a succesfull description by the excursion set analysis. A slight extension and elaboration on the original formulation enables us to frame an analytical theory explaining how the characteristic observed weblike Megaparsec scale galaxy distribution, and the equivalent frothy spatial matter distribution seen to form in computer simulations of cosmic structure formation, are natural products of a hierarchical process of gravitational clustering. This is accomplished by resorting to a complementary view of clustering evolution in which we focus on the evolution of the voids in the Megaparsec galaxy and matter distribution, spatially the dominant component (see e.g. Müller \& Maulbatsch, these proceedings). 

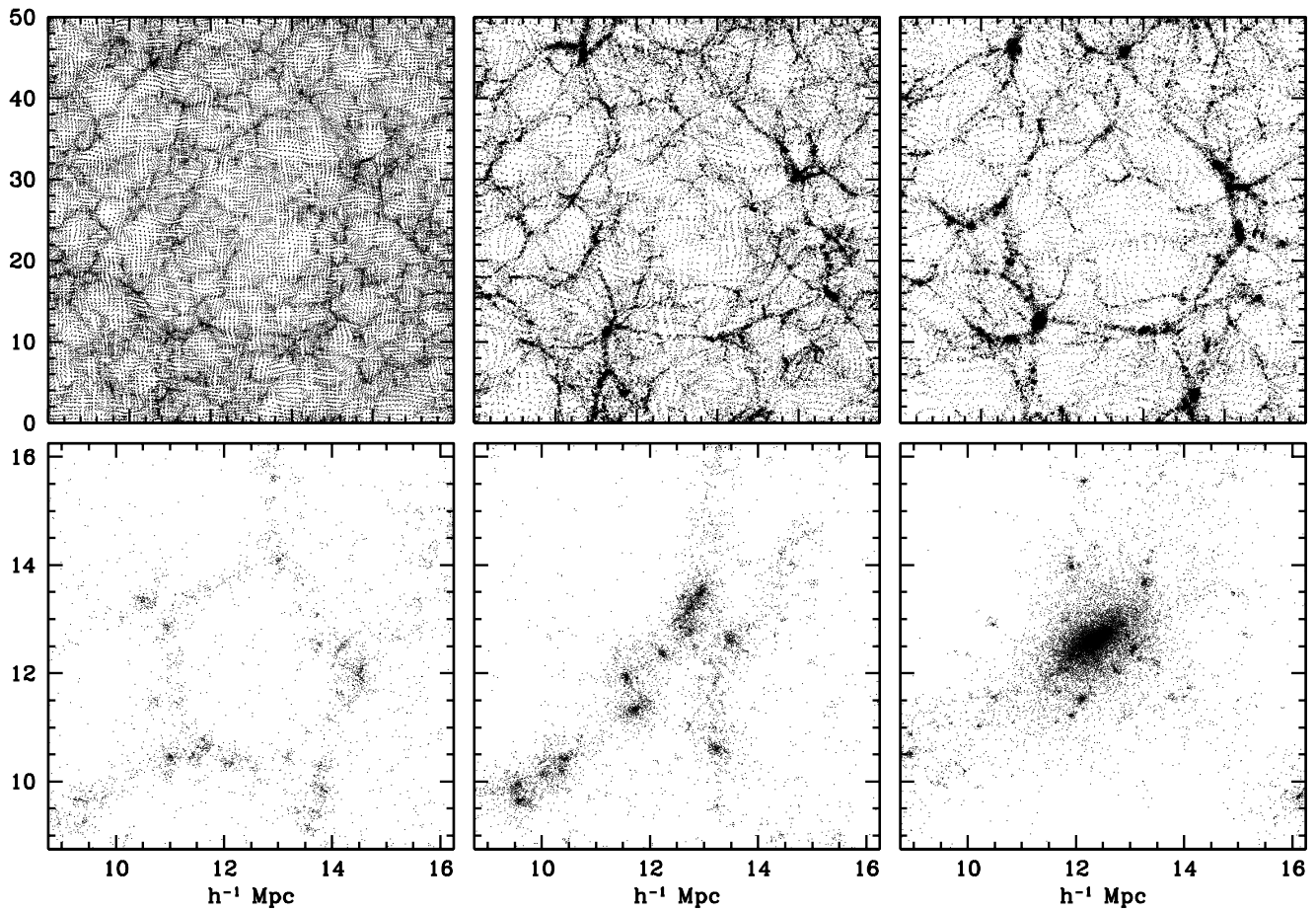

Figure 1. Illustration of the two essential "void hierarchy modes": (top) the void-in-void process, with a void growing through the merging of two or more subvoids; (bottom) the void-in-cloud process: a void demolished through the gravitational collapse of embedding region.

\section{Void evolution: the two processes}

Primordial underdensities are the progenitors of voids. Because underdensities are regions of suppressed gravitational attraction, representing an effective repulsive gravity, matter flows out of their interior and moves outward to the edges of these expanding voids. Voids expand, become increasingly empty and develop an increasingly spherical shape (Icke 1984). Matter from the void's interior piles up near the edge: usually a ridge forms around the void's rim and at a characteristic moment the void's interior shells take over the outer ones. At this shell-crossing epoch the void reaches maturity and becomes a nonlinear object expanding self-similarly, the implication being that the majority of observed voids is at or near this stage (Blumenthal et al. 1992). As voids develop from underdensities in the primordial cosmic density field, the interaction with internal substructure and external surrounding structures translates into a continuing process of hierarchical void evolution (Dubinski et al. 1993, van de Weygaert \& van Kampen 1993, Colberg et al. 2004).

The evolution of voids resembles that of dark halos in that large voids form from mergers of smaller voids that have matured at an earlier cosmic time (Fig. 1, top row). However, in contrast to dark halos, the fate of voids is ruled by two processes. Crucial is the realization that the evolving void hierarchy does not only involve the void-in-void process but also an additional aspect, the void-in-cloud process. Small voids may not only merge into larger encompassing underdensities, they may also disappear through collapse when embedded within a larger scale overdensity (Fig. 1, bottom row). In terms of the excursion set approach, it means that the one-barrier problem for the halo population has to be extended to a more complex two-barrier problem. Voids not only should ascertain themselves of having decreased their density below the void barrier $\delta_{v}$ of the shell-crossing 


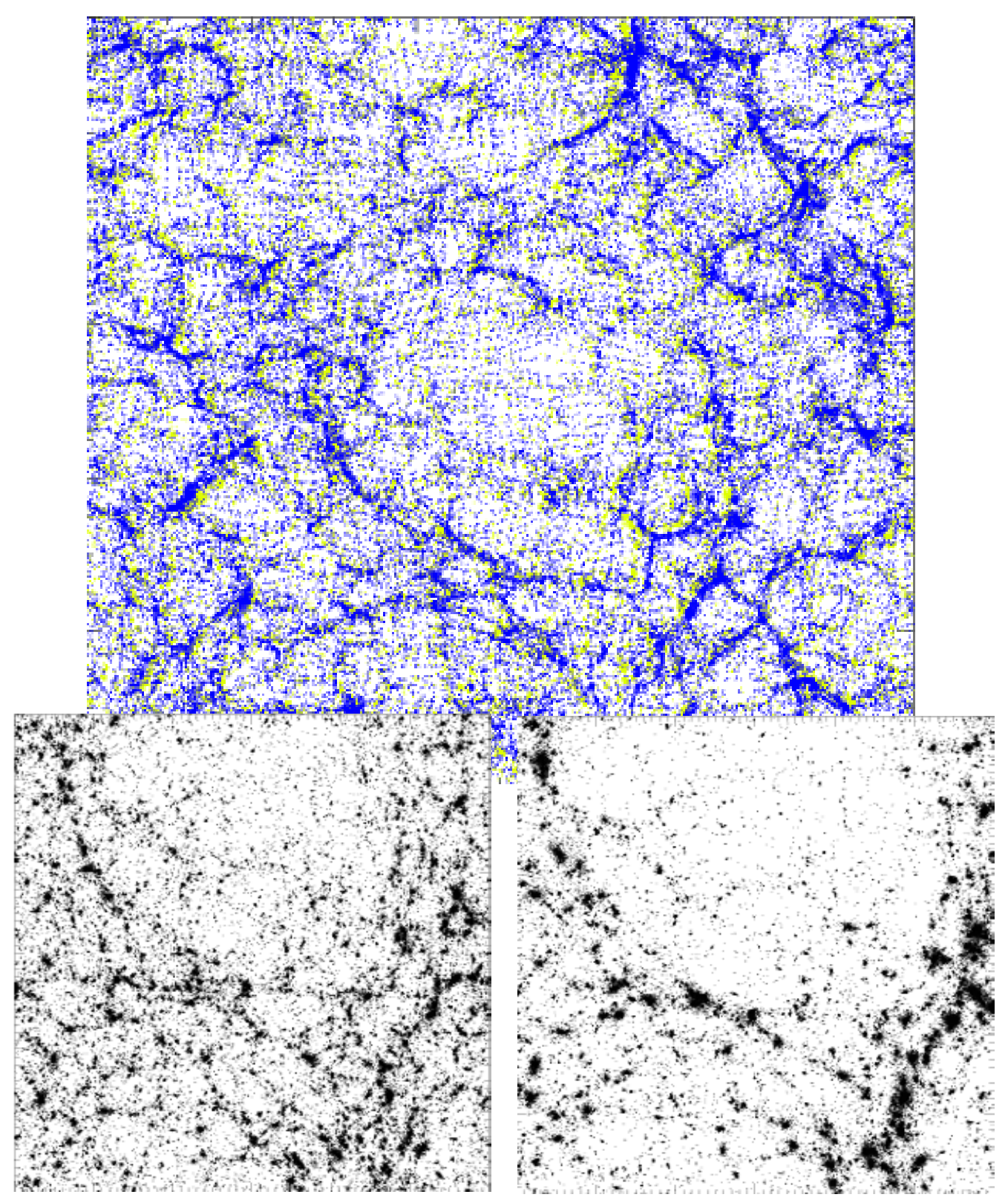

Figure 2. Identification of void collapse sites: near the boundary of an expanding voids small voids get squashed and sheared. Zoom-in on central lower region of large void (top image) at 2 different timesteps, showing the void compression process.

transition. For their survival and sheer existence it is crucial that they take into account whether they are not situated within a collapsing overdensity on a larger scale which crossed the collapse barrier $\delta_{c}$. They should follow a random walk path like type " 3 " in Fig. 3, rather than the void-in-cloud path "4"). The repercussions of this are far-reaching and it leads to a major modification of the void properties and distribution. 

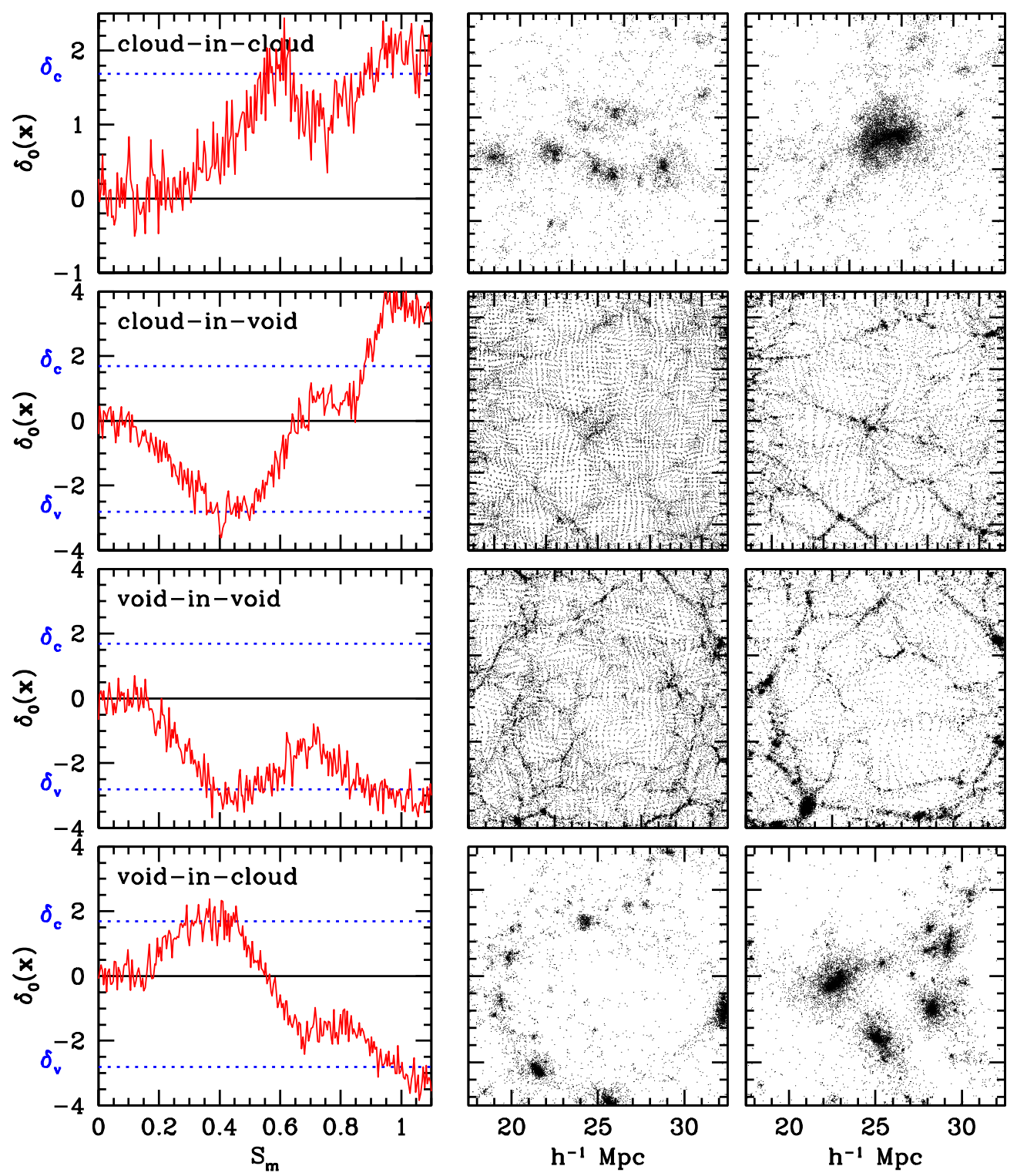

Figure 3. Four mode (extended) excursion set formalism. Each row illustrates one of the four basic modes of hierarchical clustering: the cloud-in-cloud process, cloud-in-void process, void-in-void process and void-in-cloud process (from top to bottom). Each mode is illustrated using three frames. Leftmost panels show 'random walks': the local density perturbation $\delta_{0}(\mathbf{x})$ as a function of (mass) resolution scale $S_{m}$ at an early time in an N-body simulation of cosmic structure formation. In each graph, the dashed horizontal lines indicate the collapse barrier $\delta_{\mathrm{c}}$ and the shell-crossing void barrier $\delta_{\mathrm{v}}$. The two frames on the right show how the associated particle distribution evolves. Whereas halos within voids may be observable (second row depicts a halo within a larger void), voids within collapsed halos are not (last row depicts a small void which will be squeezed to small size as the surrounding halo collapses). It is this fact which makes the calculation of void sizes qualitatively different from that usually used to estimate the mass function of collapsed halos. 


\section{Void distribution: universal, peaked and self-similar}

Analytically, the resulting expression follows by evaluating the fraction of walks which first cross $\delta_{\mathrm{v}}$ at $S$, and which do not cross $\delta_{\mathrm{c}}$ until after they have crossed $\delta_{\mathrm{v}}$ (see Fig. 3, cf. row 3 vs. row 4$)$. This distribution $\mathcal{F}\left(S, \delta_{\mathrm{v}}, \delta_{\mathrm{c}}\right)$ is given by (Sheth \& van de Weygaert 2003),

$$
S \mathcal{F}\left(S, \delta_{\mathrm{v}}, \delta_{\mathrm{c}}\right)=\sum_{j=1}^{\infty} \frac{j^{2} \pi^{2} D^{2}}{\delta_{\mathrm{v}}^{2} / S} \frac{\sin (j \pi D)}{j \pi} \times \exp \left(-\frac{j^{2} \pi^{2} D^{2}}{2 \delta_{\mathrm{v}}^{2} / S}\right),
$$

in which the relative impact of void and halo evolution on the hierarchically evolving population of voids is parameterized through $D \equiv\left|\delta_{\mathrm{v}}\right| /\left(\delta_{\mathrm{c}}-\delta_{\mathrm{v}}\right)$. A smaller value of $D$ corresponds to a diminished importance of the void-in-cloud process. The above expression may be approximated by the more accessible expression, for values of $\delta_{\mathrm{c}} /\left|\delta_{\mathrm{v}}\right| \geqslant 1 / 4$, using the "self-similar" parameter $\nu \equiv \delta_{\mathrm{v}}^{2} / S$

$$
\nu f(\nu) \approx \sqrt{\frac{\nu}{2 \pi}} \exp \left(-\frac{\nu}{2}\right) \exp \left(-\frac{\left|\delta_{\mathrm{v}}\right|}{\delta_{\mathrm{c}}} \frac{D^{2}}{4 \nu}-2 \frac{D^{4}}{\nu^{2}}\right) .
$$

The resulting distributions, for various values of $D$, are shown in Figure 4 . The void size distribution is clearly peaked about a characteristic size: the void-in-cloud mechanism is responsible for the demise of a sizeable population of small voids. The halo mass distribution diverges towards small scale masses, so that in terms of numbers the halo population is dominated by small mass objects. The void population, on the other hand, is "void" of small voids and has a sharp small-scale cut-off.

Four additional major observations readily follow from this analysis: $(\bullet)$ At any one cosmic epoch we may identify a characteristic void size which also explains why in the present-day foamlike spatial galaxy distribution voids of $\sim 20-30 h^{-1} \mathrm{Mpc}$ are the predominant feature; $(\bullet)$ The void distribution evolves self-similarly and the characteristic void size increases with time: the larger voids present at late times formed from mergers of smaller voids which constituted the dominating features at earlier epochs (Fig. 4, top

panels); $(\bullet)$ Volume integration shows that at any given time the population of voids approximately fill space, apparently squeezing the migrating high-density matter in between them; $(\bullet)$ As the size of the major share of voids will be in the order of that of the characteristic void size this observation implies that the cosmic matter distribution resembles a foamlike packing of spherical voids of approximately similar size and excess expansion rate.

To develop this generic picture into an encompassing view of the formation and evolution of the foamlike galaxy distribution various additional issues need to be addressed. One issue concerns the translation from matter into galaxy distribution, and thus the question of galaxy formation within voidlike environments. In this respect it should be noted that the suggested scale of the peaked matter distribution still falls short of that of the observed galaxy distribution (see e.g. Vogeley 2004). Another issue is the identification and location of the collapsing void population. Recent results (Fig. 2) have indicated that most of these events take place near and on the boundary of the large expanding voids: most small voids appear to get squashed and sheared (see van de Weygaert \& Babul 1996) there where the matter currents out of voids collide with the surrounding overdensities.

In all, from this study an enticing image of cosmic structure evolution emanates. A continuously evolving hierarchy of voids produces a dynamical foamlike pattern whose characteristic dimension grows continuously along with the evolution of cosmic structure, 


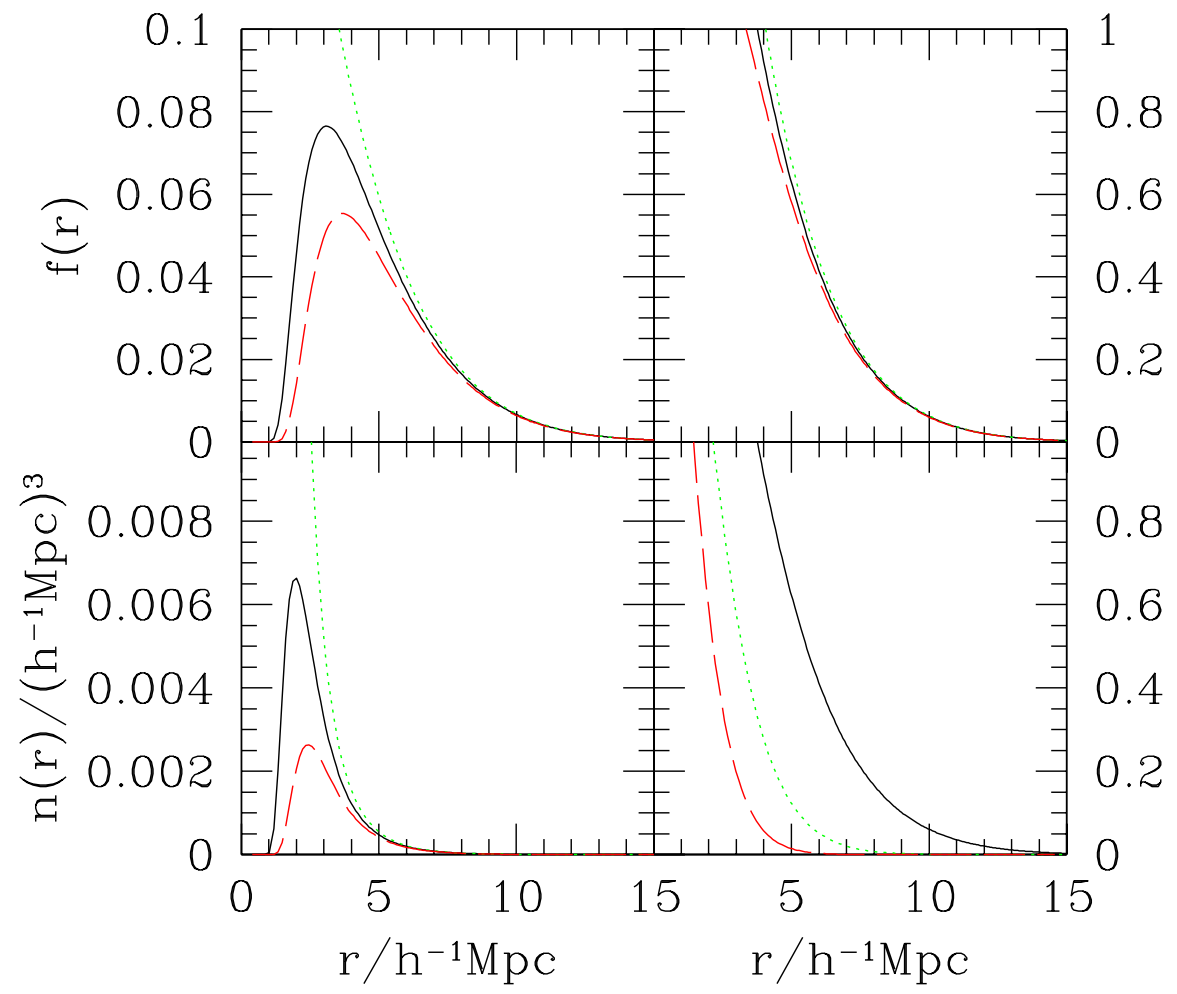

Figure 4. Distribution of predicted void radii predicted by, in an Einstein de-Sitter model with $P(k) \propto k^{-1.5}$, normalized to $\sigma_{8}=0.9$ at $z=0$. Top left panel shows the mass fraction in voids of radius $r$. Bottom left panel shows the number density of voids of radius $r$. Note that the void-size distribution is well peaked about a characteristic size provided one accounts for the void-in-cloud process. Top right panel shows the cumulative distribution of the void volume fraction. Dashed and solid curves in the top panels and bottom left panel show the two natural choices for the importance of the void-in-cloud process discussed in the text: $\delta_{\mathrm{c}}=1.06$ and 1.686 , with $\delta_{\mathrm{v}}=-2.81$. Dotted curve shows the result of ignoring the void-in-cloud process entirely. Clearly, the number of small voids decreases as the ratio of $\delta_{\mathrm{c}} /\left|\delta_{\mathrm{v}}\right|$ decreases. Bottom right panel shows the evolution of the cumulative void volume fraction distribution. The three curves in this panel are for $\delta_{\mathrm{c}}=1.686(1+z)$, where $z=0$ (solid), 0.5 (dotted) and 1 (dashed).

a Universe which at any one cosmic epoch is filled with bubbles whose size corresponds to the scale just reaching maturity.

\section{References}

Appel L., Jones B. J. T. 1990 MNRAS 245, 522

Blumenthal G.R., et al. 1992 ApJ 388, 234

Bond J. R., Cole S., Efstathiou G., Kaiser N. 1991 ApJ 379, 440

Colberg J., et al. 2004, these proceedings

Dubinski J., et al. 1993 ApJ 410, 458

Icke V. 1984 MNRAS 206, 1

Müller V., Maulbetsch C. 2004, these proceedings

Press W., Schechter P. 1974, ApJ 187, 425

Sheth R., van de Weygaert R. 2003, MNRAS, in the press

van de Weygaert R., Babul A. 1996, unpublished

van de Weygaert R., van Kampen E. 1993, MNRAS 263, 481

Vogeley M. 2004, these proceedings 